\title{
Erratum to: Unraveling the pectinolytic function of Bacteroides xylanisolvens using a RNA-seq approach and mutagenesis
}

Jordane Despres ${ }^{1}$, Evelyne Forano ${ }^{1}$, Pascale Lepercq', Sophie Comtet-Marre', Grégory Jubelin ${ }^{1}$, Carl J. Yeoman², Margret E. Berg Miller ${ }^{3}$, Christopher J. Fields ${ }^{4}$, Nicolas Terrapon ${ }^{5,6}$, Carine Le Bourvellec ${ }^{7,8}$, Catherine M.G.C. Renard ${ }^{7,8}$, Bernard Henrissat ${ }^{5,6,9}$, Bryan A. White ${ }^{3,4}$ and Pascale Mosoni ${ }^{1 *}$

\section{Erratum}

After publication of the original article [1], it came to the authors' attention that a funding source received by B. Henrissat had been accidently omitted from the Acknowledgements. The following sentence should have been included in the original article:

This work was supported in part by a grant to $\mathrm{BH}$ : European Union's Seventh Framework Program (FP/2007/2013)/ European Research Council (ERC) Grant Agreement 322820.

\begin{abstract}
Author details
'Institut National de la Recherche Agronomique (INRA), UR454 Microbiologie, Centre de Clermont-Ferrand/Theix, Saint-Genès Champanelle 63122, France.

${ }^{2}$ Department of Animal and Range Sciences, Montana State University, Bozeman, MT 59718, USA. ${ }^{3}$ Department of Animal Sciences, University of Illinois at Urbana-Champaign, Urbana, IL, USA. ${ }^{4}$ Institute for Genomic Biology, University of Illinois at Urbana-Champaign, Urbana, IL, USA. ${ }^{5}$ Architecture et Fonction des Macromolécules Biologiques (AFMB), UMR 7257 CNRS, Université Aix-Marseille, 163 Avenue de Luminy, Marseille 13288, France. ${ }^{6}$ INRA, USC 1408 AFMB, Marseille 13288, France. ${ }^{7}$ INRA, UMR408 Sécurité et Qualité des Produits d'Origine Végétale, Avignon F-84000, France. ${ }^{8}$ Université d'Avignon et des Pays de Vaucluse, UMR408 Sécurité et Qualité des Produits d'Origine Végétale, Avignon F-84000, France. ${ }^{9}$ Department of Biological Sciences, King Abdulaziz University, Jeddah, Saudi Arabia.
\end{abstract}

Published online: 06 June 2016

\section{Reference}

1. Despres J, Forano E, Lepercq P, Comtet-Marre S, Jubelin G, Yeoman CJ, et al. Unraveling the pectinolytic function of Bacteroides xylanisolvens using a RNA-seq approach and mutagenesis. BMC Genomics. 2016;27:17. doi:10. 1186/s12864-016-2472-1.

\footnotetext{
* Correspondence: pascale.mosoni@clermont.inra.fr

'Institut National de la Recherche Agronomique (INRA), UR454 Microbiologie, Centre de Clermont-Ferrand/Theix, Saint-Genès Champanelle 63122, France
} 